\title{
Improving salinity tolerance in transplanted aman rice (Oryza sativa L.) by exogenous application of proline
}

\author{
Noor-Us-Sane ${ }^{1}$, Dhiman Bhusan ${ }^{1}$, Papon Kumar Deb Nath ${ }^{2}$, Yoshiyuki Murata ${ }^{3}$ and ${ }^{\bowtie d}$ Md. Anamul \\ Hoque $^{1}$ \\ ${ }^{1}$ Department of Soil Science, Bangladesh Agricultural University, Mymensingh-2202, Bangladesh \\ ${ }^{2}$ Department of Environmental Science, Bangladesh Agricultural University, Mymensingh-2202, Bangladesh \\ ${ }^{3}$ Graduate School of Environmental and Life Science, Okayama University, Okayama-8530, Japan
}

\begin{tabular}{l}
\hline ARTICLE INFO OPENCACCESS \\
\hline Article history: \\
Received : 05 March 2019 \\
Accepted : 29 May 2019 \\
Published: 30 June 2019 \\
\hline Keywords: \\
Proline, salt stress, nutrient \\
uptake, $\mathrm{K}^{+} / \mathrm{Na}^{+}$ratio, aman rice \\
\hline Correspondence: \\
Md. Anamul Hoque \\
\: anamul71@bau.edu.bd
\end{tabular}

\section{Abstract}

Salinity is the major factor reducing crop yield in coastal areas of Bangladesh. Proline (Pro) application with suitable crop varieties having higher yield potential could contribute to the improvement of crop production in saline areas. The main objective of this study was to investigate the mitigation of adverse effects of salinity in aman rice by exogenously applied Pro. The experiment was carried out at the farmer's field of Batiaghata, Khulna. Characteristically, the soil was silty clay loam having $\mathrm{pH} 6.7, \mathrm{EC} 4.6 \mathrm{dS} \mathrm{m}^{-1}$, CEC 23 meq/100 g soil, organic matter $0.71 \%$. Rice (Oryza sativa $\mathrm{L}$.) variety BR23 was used as plant material. The experiment was laid out in a randomized complete block design with three replications. There were different treatment combinations namely control (no Pro), $25 \mathrm{mM}$ Pro at seedling stage, 25 $\mathrm{mM}$ Pro at vegetative stage, $25 \mathrm{mM}$ Pro at seedling and vegetative stages, $50 \mathrm{mM}$ Pro at seedling stage, 50 $\mathrm{mM}$ Pro at vegetative stage, $50 \mathrm{mM}$ Pro at seedling and vegetative stages, $100 \mathrm{mM}$ Pro at seedling stage, $100 \mathrm{mM}$ Pro at vegetative stages, and $100 \mathrm{mM}$ Pro at seedling and vegetative stages. Recommended doses of N, P, K, S and Zn fertilizers were applied to the all experimental plots. Thirty-day-old seedlings were transplanted in the experimental plots. Proline solutions were sprayed over plant leaves with the help of sprayer as per treatments. Salinity caused significant reductions in growth and yield of BR23 by decreasing plant height, number of effective tillers, panicle length, filled grains panicle ${ }^{-1}$ and 1000 -grain weight. On the other hand, exogenous application of Pro showed a significant increase in growth and yield of BR23 under saline conditions. Results also revealed that growth and yield of rice did not increase proportionally with the increasing doses of Pro. Proline application resulted in significant increases in $\mathrm{K}^{+} / \mathrm{Na}^{+}$and nutrient uptake by rice under salinity. The present study suggests that exogenous application of Pro confers tolerance to salinity in aman rice by increasing $\mathrm{K}^{+} / \mathrm{Na}^{+}$ratio and nutrient uptake.
\end{abstract}

(C2019 by authors and BAURES. This work is licensed under the Creative Commons Attribution International License (CC By 4.0).

\begin{abstract}
Copyright:
(c) (i)

\section{Introduction}

Growth and yield reduction of crops are the serious issue in salinity prone areas of the world. About $20 \%$ of world's cultivated areas and nearly half of the world's irrigated lands are affected by salinity (Mali et al., 2012). Salinity effects are more conspicuous in arid and semiarid regions where limited rainfall, high evapotranspiration and high temperature associated with poor water and soil management (Azevedo et al., 2006; Jaleel et al., 2008). Agricultural productivity is severely affected by soil salinity, and the damaging effect of salt accumulation in agricultural soils has become an important environmental concern (Jaleel et al., 2007).
\end{abstract}

The world population is increasing rapidly and may reach 6 to 9.3 billion by the year 2050 whereas the crop production is decreasing rapidly because of the negative impact of various environmental stresses; therefore, it is now very important to develop stress-tolerant varieties to cope with this upcoming problem of food security. In addition, increased salinity of arable land is expected to have devastating global effects, resulting in up to $50 \%$ Cite this article

Sane, N.U., Bhusan, D., Nath, P.K.D., Murata, Y. and Hoque, M.A. 2019. Improving salinity tolerance in transplanted aman rice (Oryza sativa L.) by exogenous application of proline. Journal of Bangladesh Agricultural University, 17(1): 194-199. https://doi.org/10.3329/jbau.v17i2.41943 land losses by the middle of the twenty-first century (Mahajan and Tuteja, 2005).

Salinity causes unfavorable environment and hydrological situation that restrict the normal crop production throughout the year (Haque et al., 2014). The factors which contribute significantly to the development of saline soil are tidal flooding during wet season (June-October), direct inundation by saline water, and upward or lateral movement of saline ground water during dry season (November-May). Rice is mainly grown in salinity affected areas of Bangladesh but the average yield is very low due to lack of salt-tolerant high yielding variety and inappropriate management practices. In Bangladesh, out of 2.85 million hectares of the coastal and off-shore areas, about 1.06 million hectares are affected by salinity (SRDI, 2010).

Plants possess different defense mechanisms in order to cope with stress; one of them is associated with accumulation of osmoprotectants. Proline is one of the major osmoprotectant osmolytes, which is synthesized 
by many plants in response to stress including salinity, thereby maintaining the osmotic status of the cell to ameliorate the abiotic stress (Chinnusamy et al., 2005; Burritt, 2012; Hossain et al., 2014). Proline plays roles in scavenging free radicals, stabilizing subcellular structures, and buffering celluler redox potential under stresses (Hoque et al., 2008; Banu et al., 2009; Hossain et al., 2014). Exogenous application of Pro also provides osmoprotection and facilitates the growth of salinitystress plants. Proline can protect cells against salinityinduced oxidative stress by up-regulating activities of various antioxidant enzyme activities (Hoque et al., 2007; Bhusan et al., 2016).

Exogenous application of Pro already gained considerable attention in mitigating the adverse effect of salt stress (Ashraf and Foolad, 2007; Hoque et al., 2007). There are increasing evidences that Pro application effectively regulates osmotic potential and plays a vital role in sustaining plant growth under osmotic stress (Ali et al., 2007; Ashraf and Foolad, 2007; Hoque et al., 2007). Exogenous Pro enhanced the antioxidant enzymatic activities viz. ascorbate peroxidase, peroxidase and catalase during both stress and recovery period in rice (Nounjan et al., 2012; Bhusan et al., 2016). Little information is available on the beneficial roles of exogenous Pro in modulating salt stress tolerance in rice at various phases of plant growth. Therefore, the present study aimed to investigate the mitigation of the adverse effects of salinity in aman rice by exogenously applied Pro at both seedling and vegetative stages.

\section{Materials and Methods}

\section{Experimental site and soils}

The field experiment was carried out at the farmer's field of Batiaghata, Khulna belongs to the Agroecological Zone of the Ganges Tidal Floodplain (AEZ 13). Characteristically, the soil was silty clay loam having $\mathrm{pH}$ 6.7, EC $4.6 \mathrm{dS} \mathrm{m}{ }^{-1}$, CEC $23 \mathrm{meq} / 100 \mathrm{~g}$ soil and organic matter $0.71 \%$. The experimental area is included into the tropical monsoon climate. There are three monsoon periods appear in this region. The monsoon period lasts from May to October. About 88\% of the total rainfall is observed in this time. Hailstone also occurs during this time. Sometimes storms locally called Kalboishakhi are observed.

\section{Plant materials and treatments}

The experiment was laid out in a randomized complete block design with three replications. Rice variety BR23 was used as plant material. There were ten treatment combinations with different concentrations of Pro at seedling and vegetative stages like as $\mathrm{T}_{0}=$ Control (no Pro), $\mathrm{T}_{1}=25 \mathrm{mM}$ Pro at seedling stage, $\mathrm{T}_{2}=25 \mathrm{mM}$ Pro at vegetative stage, $T_{3}=25 \mathrm{mM}$ Pro at seedling and vegetative stages, $T_{4}=50 \mathrm{mM}$ Pro at seedling stage, $T_{5}$ $=50 \mathrm{mM}$ Pro at vegetative stage, $\mathrm{T}_{6}=50 \mathrm{mM}$ Pro at seedling and vegetative stages, $\mathrm{T}_{7}=100 \mathrm{mM}$ Pro at seedling stage, $\mathrm{T}_{8}=100 \mathrm{mM}$ Pro at vegetative stage, and $\mathrm{T}_{9}=100 \mathrm{mM}$ Pro at seedling and vegetative stages. Recommended doses of TSP, MoP, gypsum, and zinc sulphate were applied to all the experimental plots (4.0 $\mathrm{m} \times 2.5 \mathrm{~m}$ ) during final land preparation. Recommended dose of urea was applied in three splits. Thirty-day-old rice seedlings were transplanted in the experimental plots. Three seedlings per hill were placed at a spacing of $25 \mathrm{~cm} \times 20 \mathrm{~cm}$. Proline solutions were sprayed over plant leaves with the help of sprayer. The volume of the spray was $25 \mathrm{ml}$ per plant at both seedling and vegetative stages. Tween-20 was used as a sticky substance which helps Pro solution's droplet, maintaining a close contact with plant leaves. Other intercultural operations were done when necessary. Maturity of crop was determined when about $90 \%$ grains became golden yellow. The crop was harvested at full maturity.

\section{Chemical analysis of plant samples}

The representative grain and straw samples were dried in an oven at $65^{\circ} \mathrm{C}$ for about 24 hours before they were ground by a grinding machine. The prepared samples were stored in paper bags and finally kept into desiccators until analysis. The $\mathrm{N}, \mathrm{P}, \mathrm{K}, \mathrm{S}$ and $\mathrm{Na}$ contents from grain and straw samples were determined following standard method as described by Khanam et al. (2001).

\section{Statistical analysis}

Data were analyzed statistically using analysis of variance with the help of software package MSTAT-C. The significant differences between mean values were compared by Duncan's Multiple Range Test. Differences at $P \leq 0.05$ were considered significant.

\section{Results and Discussion}

\section{Growth and yield components of rice}

Salinity caused a significant decrease in plant height whereas Pro application significantly increased plant height (Table 1). Salinity significantly decreased effective tillers hill ${ }^{-1}$ of BR23. All treatments except 100 $\mathrm{mM}$ Pro at seedling stage increased effective tillers per hill over control. It was observed that there were no significant variations in panicle length among the treatments that exposed varying concentration of Pro application such as $25 \mathrm{mM}, 50 \mathrm{mM}, 100 \mathrm{mM}$ Pro at seedling stage, vegetative stage, and both seedling and vegetative stages. Salinity caused a drastic decrease in filled grains per panicle of BR23. Exogenous application of Pro at different growth stages significantly increased filled grains per panicle under salinity conditions. Table 1 also shows that salinity significantly reduced the 1000grain weight of BR23. All the Pro treatments increased 1000-grain weight over control. Some of the Pro treatments contributed to the significant increase of 1000-grain weight under saline condition (Table 1). 
Table 1. Effect of exogenous proline on the growth of T. Aman rice (BR23) under salinity conditions

\begin{tabular}{|c|c|c|c|c|c|}
\hline Treatment & $\begin{array}{l}\text { Plant height } \\
(\mathrm{cm})\end{array}$ & $\begin{array}{c}\text { No. of effective } \\
\text { tillers/ } \\
\text { Hill } \\
\end{array}$ & $\begin{array}{l}\text { Panicle } \\
\text { length } \\
(\mathrm{cm})\end{array}$ & $\begin{array}{c}\text { No. of filled } \\
\text { grains/ } \\
\text { panicle }\end{array}$ & $\begin{array}{c}\text { 1000-grain } \\
\text { weight } \\
(\mathrm{gm})\end{array}$ \\
\hline $\mathrm{T}_{0}$ Control & $119 \mathrm{c}$ & $12 \mathrm{c}$ & $24 \mathrm{a}$ & $88 b$ & $21.24 \mathrm{~d}$ \\
\hline $\mathrm{T}_{1} 25 \mathrm{mM}$ proline at seedling stage & $126 a b c$ & $15 \mathrm{a}$ & $24 \mathrm{a}$ & $109 \mathrm{a}$ & 22.40abcd \\
\hline $\mathrm{T}_{2} 25 \mathrm{mM}$ proline at vegetative stage & $122 \mathrm{bc}$ & $13 \mathrm{bc}$ & $24 \mathrm{a}$ & $112 \mathrm{a}$ & 22.61abcd \\
\hline $\mathrm{T}_{3} 25 \mathrm{mM}$ proline at seedling and vegetative stages & $125 \mathrm{abc}$ & $14 \mathrm{ab}$ & $24 \mathrm{a}$ & $113 \mathrm{a}$ & $22.33 \mathrm{abcd}$ \\
\hline $\mathrm{T}_{4} 50 \mathrm{mM}$ proline at seedling stage & $129 \mathrm{ab}$ & $13 \mathrm{bc}$ & $23 b$ & $103 \mathrm{a}$ & $23.37 \mathrm{ab}$ \\
\hline $\mathrm{T}_{5} 50 \mathrm{mM}$ proline at vegetative stage & $128 \mathrm{ab}$ & $13 \mathrm{bc}$ & $24 \mathrm{a}$ & $101 \mathrm{a}$ & $23.52 \mathrm{a}$ \\
\hline $\mathrm{T}_{6} 50 \mathrm{mM}$ proline at seedling and vegetative stages & $129 \mathrm{ab}$ & $14 \mathrm{ab}$ & $23 b$ & $107 \mathrm{a}$ & 22.28abcd \\
\hline $\mathrm{T}_{7} 100 \mathrm{mM}$ proline at seedling stage & $123 \mathrm{abc}$ & $12 \mathrm{c}$ & $24 \mathrm{a}$ & $104 \mathrm{a}$ & $22.74 \mathrm{abc}$ \\
\hline $\mathrm{T}_{8} 100 \mathrm{mM}$ proline at vegetative stage & $130 \mathrm{a}$ & $14 \mathrm{ab}$ & $24 \mathrm{a}$ & $104 \mathrm{a}$ & $22.01 \mathrm{bcd}$ \\
\hline $\mathrm{T}_{9} 10 \mathrm{mM}$ proline at seedling and vegetative stages & $128 \mathrm{ab}$ & $14 \mathrm{ab}$ & $24 \mathrm{a}$ & $106 \mathrm{a}$ & $21.89 \mathrm{~cd}$ \\
\hline $\mathrm{SE}( \pm)$ & 1.140 & 0.306 & 0.133 & 2.221 & 0.214 \\
\hline $\mathrm{CV}(\%)$ & 3.05 & 5.02 & 1.75 & 6.19 & 3.19 \\
\hline
\end{tabular}

Same letter in a column represents insignificant difference at $\mathrm{p}<0.05$.

$\mathrm{SE}=$ Standard errors of means

$\mathrm{CV}=$ Co-efficient of variation

A large body of evidences reports that salinity has negative impact on growth and yield components of crops. Momayezi et al. (2010) showed that number of effective tillers per hill was decreased by increasing salt level in rice. Islam et al. (2011) on hybrid rice and Miah et al. (1992) on two rice varieties found that plant height decreased with increasing salinity. Papon et al. (2015) showed that salt stress reduced filled grains of both saltsensitive and salt-tolerant rice varieties. On the other hand, Abbas et al. (2012) demostrated that exogenous application of Pro increased plant height of Citrus sinensis (L.) under $\mathrm{NaCl}$ stress. Deivanai et al. (2011) also showed that Pro application increased plant height in two Malaysian rice cultivars (MR220 and MR232). Papon et al. (2015) showed that Pro application increased number of filled grains per panicle under saline conditions. Recently it is also reported by Bhusan et al. (2016) that exogenous Pro application increased growth of rice.

\section{Grain and straw yields of rice}

Plant exposed with salinity significantly decreased grain yield of BR23 rice (Table 2). Foliar application of Pro over plant leaves significantly increased grain yield under salinity condition. All the treatments increased grain yield over control. Straw yield of BR23 was drastically reduced due to salinity. All the Pro treatments significantly increased straw yield over control (Table 2).

Table 2. Effect of exogenous proline on the grain and straw yields of T. Aman rice (BR23) under salinity conditions

\begin{tabular}{lcc}
\hline Treatment & Grain yield (kg/ha) & Straw yield (kg/ha) \\
\hline $\mathrm{T}_{0}$ Control & $4672 \mathrm{c}$ & $5063 \mathrm{e}$ \\
$\mathrm{T}_{1} 25 \mathrm{mM}$ proline at seedling stage & $5438 \mathrm{a}$ & $5863 \mathrm{abc}$ \\
$\mathrm{T}_{2} 25 \mathrm{mM}$ proline at vegetative stage & $4980 \mathrm{ab}$ & $5929 \mathrm{ab}$ \\
$\mathrm{T}_{3} 25 \mathrm{mM}$ proline at seedling and vegetative stages & $5363 \mathrm{ab}$ & $6329 \mathrm{a}$ \\
$\mathrm{T}_{4} 50 \mathrm{mM}$ proline at seedling stage & $5016 \mathrm{ab}$ & $5929 \mathrm{ab}$ \\
$\mathrm{T}_{5} 50 \mathrm{mM}$ proline at vegetative stage & $4859 \mathrm{bc}$ & $5130 \mathrm{de}$ \\
$\mathrm{T}_{6} 50 \mathrm{mM}$ proline at seedling and vegetative stages & $5361 \mathrm{ab}$ & $5862 \mathrm{abc}$ \\
$\mathrm{T}_{7} 100 \mathrm{mM}$ proline at seedling stage & $4924 \mathrm{ab}$ & $5663 \mathrm{bcd}$ \\
$\mathrm{T}_{8} 100 \mathrm{mM}$ proline at vegetative stage & $4907 \mathrm{~b}$ & $5163 \mathrm{de}$ \\
$\mathrm{T}_{9} 10 \mathrm{mM}$ proline at seedling and vegetative stages & $4941 \mathrm{ab}$ & $5297 \mathrm{de}$ \\
\hline $\mathrm{SE}( \pm)$ & 80.188 & 136.486 \\
$\mathrm{CV}(\%)$ & 5.37 & 5.56 \\
\hline
\end{tabular}

Same letter in a column represents insignificant difference at $\mathrm{p}<0.05$.

$\mathrm{SE}=$ Standard errors of means

$\mathrm{CV}=$ Co-efficient of variation

Miah et al. (1992) on two rice varieties found that salinity decreased straw yield of rice at 2.4, 6.0 and 11.8 $\mathrm{dSm}^{-1}$ conditions. It has been demonstrated that salt stress reduced grain and straw yields of rice (Bhusan et al., 2016). The protective mechanisms of Pro have been increasingly reported in the literature in plants against various stresses. It has been reported that exogenous Pro application increased grain and straw yields of rice (Papon et al., 2015; Bhusan et al., 2016).

\section{Nutrient content and uptake}

N content and uptake: There were significant variations in rice grain $\mathrm{N}$ content due to application of Pro. Most of the Pro treatments increased N content over control (Table 3). There was also significant variation in straw N content due to application of Pro. Most of the Pro treatments resulted in lower straw $\mathrm{N}$ content than control (Table 3). There were significant variations in 
rice total $\mathrm{N}$ uptake with application of Pro. All the treatments increased total $\mathrm{N}$ uptake over control (Table 3).

$P$ content and uptake: There were significant variations in rice grain $\mathrm{P}$ content due to exogenously applied Pro. Most of the Pro treatments increased $\mathrm{P}$ content over control (Table 4). Significant variations in rice straw $\mathrm{P}$ content were also observed in response to Pro application. All the treatments increased P content over control (Table 4). There were significant variations in rice total $\mathrm{P}$ uptake with application of Pro. All the treatments increased total $\mathrm{P}$ uptake over control (Table 4).

Table 3. Effect of exogenous proline on nitrogen content and uptake by T. Aman rice (BR23) under salinity conditions

\begin{tabular}{|c|c|c|c|c|c|}
\hline \multirow[t]{2}{*}{ Treatment } & \multicolumn{2}{|c|}{ N Content \% } & \multicolumn{3}{|c|}{ N uptake $(\mathrm{kg} / \mathrm{ha})$} \\
\hline & Grain & Straw & Grain & Straw & Total \\
\hline $\mathrm{T}_{0}$ Control & $0.920 \mathrm{e}$ & $0.53 \mathrm{~b}$ & $43.17 \mathrm{f}$ & $26.93 \mathrm{~cd}$ & $70.10 \mathrm{ef}$ \\
\hline $\mathrm{T}_{1} 25 \mathrm{mM}$ proline at seedling stage & $1.148 b c d$ & $0.44 \mathrm{~cd}$ & $62.43 b$ & $26.27 \mathrm{~cd}$ & $88.70 \mathrm{bc}$ \\
\hline $\mathrm{T}_{2} 25 \mathrm{mM}$ proline at vegetative stage & $1.400 \mathrm{a}$ & $0.42 \mathrm{~d}$ & $69.72 \mathrm{a}$ & 24.90de & $94.62 \mathrm{a}$ \\
\hline $\mathrm{T}_{3} 25 \mathrm{mM}$ proline at seedling and vegetative stages & $1.06 \mathrm{cde}$ & $0.42 \mathrm{~d}$ & $57.06 \mathrm{~cd}$ & $26.58 \mathrm{~cd}$ & $83.64 \mathrm{c}$ \\
\hline $\mathrm{T}_{4} 50 \mathrm{mM}$ proline at seedling stage & $1.20 \mathrm{bc}$ & $0.50 \mathrm{bc}$ & $60.36 \mathrm{bc}$ & $29.88 b$ & $90.27 \mathrm{ab}$ \\
\hline $\mathrm{T}_{5} 50 \mathrm{mM}$ proline at vegetative stage & $0.920 \mathrm{e}$ & $0.42 \mathrm{~d}$ & $54.90 \mathrm{f}$ & $22.55 f$ & $76.45 f$ \\
\hline $\mathrm{T}_{6} 50 \mathrm{mM}$ proline at seedling and vegetative stages & $1.09 \mathrm{~cd}$ & $0.47 \mathrm{bcd}$ & $58.54 \mathrm{c}$ & $27.90 \mathrm{bc}$ & $86.44 \mathrm{bc}$ \\
\hline $\mathrm{T}_{7} 100 \mathrm{mM}$ proline at seedling stage & $1.26 \mathrm{~b}$ & $0.588 \mathrm{a}$ & $62.04 \mathrm{~b}$ & $33.29 \mathrm{a}$ & $95.33 \mathrm{a}$ \\
\hline $\mathrm{T}_{8} 100 \mathrm{mM}$ proline at vegetative stage & $1.12 \mathrm{bcd}$ & $0.42 \mathrm{~d}$ & $54.96 \mathrm{~d}$ & $21.68 \mathrm{f}$ & $77.92 d$ \\
\hline $\mathrm{T}_{9} 10 \mathrm{mM}$ proline at seedling and vegetative stages & $1.03 \mathrm{de}$ & $0.44 \mathrm{~cd}$ & $51.19 \mathrm{e}$ & $23.73 \mathrm{ef}$ & 74.92de \\
\hline $\mathrm{SE}( \pm)$ & 0.046 & 0.018 & 3.42 & 5.12 & 3.49 \\
\hline $\mathrm{CV}(\%)$ & 7.05 & 7.90 & 2.58 & 1.14 & 3.18 \\
\hline
\end{tabular}

Same letter in a column represents insignificant difference at $\mathrm{p}<0.05$.

$\mathrm{SE}=$ Standard errors of means

$\mathrm{CV}=\mathrm{Co}$-efficient of variation

Table 4. Effect of exogenous proline on phosphorus content and uptake by T. Aman rice (BR23) under salinity conditions

\begin{tabular}{lccccc}
\hline Treatment & \multicolumn{2}{c}{ P Content $\%$} & \multicolumn{3}{c}{ P uptake $(\mathrm{kg} / \mathrm{ha})$} \\
\cline { 2 - 5 } & Grain & Straw & Grain & Straw & Total \\
\hline $\mathrm{T}_{0}$ Control & $0.18 \mathrm{~d}$ & $0.06 \mathrm{c}$ & $8.40 \mathrm{f}$ & $3.04 \mathrm{f}$ & $11.44 \mathrm{~g}$ \\
$\mathrm{~T}_{1} 25 \mathrm{mM}$ proline at seedling stage & $0.26 \mathrm{a}$ & $0.11 \mathrm{a}$ & $14.14 \mathrm{a}$ & $6.45 \mathrm{a}$ & $20.59 \mathrm{a}$ \\
$\mathrm{T}_{2} 25 \mathrm{mM}$ proline at vegetative stage & $0.18 \mathrm{~d}$ & $0.09 \mathrm{~b}$ & $8.96 \mathrm{def}$ & $5.34 \mathrm{~cd}$ & $14.30 \mathrm{e}$ \\
$\mathrm{T}_{3} 25 \mathrm{mM}$ proline at seedling and vegetative stages & $0.20 \mathrm{c}$ & $0.09 \mathrm{~b}$ & $10.73 \mathrm{c}$ & $5.69 \mathrm{bc}$ & $16.42 \mathrm{c}$ \\
$\mathrm{T}_{4} 50 \mathrm{mM}$ proline at seedling stage & $0.15 \mathrm{e}$ & $0.09 \mathrm{~b}$ & $7.52 \mathrm{~g}$ & $5.33 \mathrm{~cd}$ & $12.85 \mathrm{f}$ \\
$\mathrm{T}_{5} 50 \mathrm{mM}$ proline at vegetative stage & $0.26 \mathrm{a}$ & $0.10 \mathrm{ab}$ & $12.63 \mathrm{~b}$ & $5.13 \mathrm{~d}$ & $17.76 \mathrm{~b}$ \\
$\mathrm{~T}_{6} 50 \mathrm{mM}$ proline at seedling and vegetative stages & $0.23 \mathrm{~b}$ & $0.10 \mathrm{ab}$ & $12.33 \mathrm{~b}$ & $5.86 \mathrm{~b}$ & $18.19 \mathrm{~b}$ \\
$\mathrm{~T}_{7} 100 \mathrm{mM}$ proline at seedling stage & $0.18 \mathrm{~d}$ & $0.10 \mathrm{ab}$ & $8.86 \mathrm{ef}$ & $5.66 \mathrm{bc}$ & $14.52 \mathrm{de}$ \\
$\mathrm{T}_{8} 100 \mathrm{mM}$ proline at vegetative stage & $0.20 \mathrm{c}$ & $0.090 \mathrm{~b}$ & $9.81 \mathrm{~d}$ & $4.65 \mathrm{e}$ & $14.46 \mathrm{de}$ \\
$\mathrm{T}_{9} 10 \mathrm{mM}$ proline at seedling and vegetative stages & $0.19 \mathrm{~cd}$ & $0.11 \mathrm{a}$ & $9.38 \mathrm{de}$ & $5.82 \mathrm{~b}$ & $15.20 \mathrm{~d}$ \\
\hline $\mathrm{SE}( \pm)$ & 7.94 & 10.64 & 4.76 & 4.68 & 3.10 \\
$\mathrm{CV}(\%)$ & 0.011 & 0.0045 & 0.673 & 0.293 & 0.857 \\
\hline
\end{tabular}

Same letter in a column represents insignificant difference at $\mathrm{p}<0.05$.

$\mathrm{SE}=$ Standard errors of means

$\mathrm{CV}=\mathrm{Co}$-efficient of variation

$S$ content and uptake: Significant variation in grain $S$ content was observed due to different treatments. Surprisingly, most of the Pro treatments did not result in increase in grain $\mathrm{S}$ content (Table 5). There were no significant variations in straw $\mathrm{S}$ content with application of Pro. Most of the treatments were found to be lower straw S content than control. Moreover, all the treatments except $25 \mathrm{mM}$ Pro at seedling and vegetative stages were also found to be lower $S$ uptake by straw than control (Table 5). There were significant variations in total S uptake due to different treatment combinations. In some cases, exogenous Pro increased total $\mathrm{S}$ uptake but not remarkably (Table 5).
Nutrients such as $\mathrm{N}, \mathrm{P}, \mathrm{K}$ and $\mathrm{S}$ play essential roles in plant metabolism. There are evidences that Pro minimizes the adverse effects of various stresses on plants by affecting the uptake and accumulation of inorganic nutrients (Ali et al., 2008). Similar to our results, Abd El-Samad et al. (2011) showed that application of Pro increased NPK nutrient uptake in rice plants.

\section{$\mathrm{K}^{+} / \mathrm{Na}^{+}$ratio in grain}

Potassium and sodium ratio in rice grain significantly decreased due to salinity while application of Pro significantly ameliorated $\mathrm{K}^{+} / \mathrm{Na}^{+}$ratio. All the treatments increased $\mathrm{K}^{+} / \mathrm{Na}^{+}$ratio over control. The 
$\mathrm{K}^{+} / \mathrm{Na}^{+}$ratio was found to be highest at $50 \mathrm{mM}$ Pro application at seedling and vegetative stages (Table 6).

\section{$\mathrm{K}^{+} / \mathrm{Na}^{+}$ratio in straw}

The $\mathrm{K}^{+} / \mathrm{Na}^{+}$ratio in rice straw significantly decreased due to salinity but application of Pro significantly increased the $\mathrm{K}^{+} / \mathrm{Na}^{+}$ratio. All the treatments increased $\mathrm{K}^{+} / \mathrm{Na}^{+}$ratio over control. The $\mathrm{K}^{+} / \mathrm{Na}^{+}$ratio was found to be highest at $25 \mathrm{mM}$ Pro application at vegetative stage (Table 6).

Table 5. Effect of exogenous proline on sulphur content and uptake by T. Aman rice (BR23) under salinity conditions

\begin{tabular}{lccccc}
\hline Treatment & \multicolumn{2}{c}{$\mathrm{S}$ Content $\%$} & \multicolumn{3}{c}{ S uptake (kg/ha) } \\
\cline { 2 - 6 } & Grain & Straw & Grain & Straw & Total \\
\hline $\mathrm{T}_{0}$ Control & $0.09 \mathrm{a}$ & $0.14 \mathrm{a}$ & $4.58 \mathrm{ab}$ & $7.29 \mathrm{~b}$ & $11.87 \mathrm{abcd}$ \\
$\mathrm{T}_{1} 25 \mathrm{mM}$ proline at seedling stage & $0.07 \mathrm{~cd}$ & $0.10 \mathrm{e}$ & $4.02 \mathrm{~cd}$ & $6.38 \mathrm{c}$ & $10.40 \mathrm{ef}$ \\
$\mathrm{T}_{2} 25 \mathrm{mM}$ proline at vegetative stage & $0.09 \mathrm{a}$ & $0.12 \mathrm{abcde}$ & $4.84 \mathrm{ab}$ & $7.59 \mathrm{ab}$ & $12.43 \mathrm{ab}$ \\
$\mathrm{T}_{3} 25 \mathrm{mM}$ proline at seedling and vegetative stages & $0.08 \mathrm{bc}$ & $0.13 \mathrm{abcd}$ & $4.50 \mathrm{~b}$ & $8.25 \mathrm{a}$ & $12.75 \mathrm{a}$ \\
$\mathrm{T}_{4} 50 \mathrm{mM}$ proline at seedling stage & $0.07 \mathrm{bcd}$ & $0.12 \mathrm{bcde}$ & $3.79 \mathrm{~cd}$ & $7.38 \mathrm{~b}$ & $11.17 \mathrm{de}$ \\
$\mathrm{T}_{5} 50 \mathrm{mM}$ proline at vegetative stage & $0.08 \mathrm{~b}$ & $0.11 \mathrm{de}$ & $4.12 \mathrm{c}$ & $5.79 \mathrm{c}$ & $9.91 \mathrm{f}$ \\
$\mathrm{T}_{6} 50 \mathrm{mM}$ proline at seedling and vegetative stages & $0.07 \mathrm{~d}$ & $0.12 \mathrm{abcde}$ & $3.73 \mathrm{~d}$ & $7.52 \mathrm{ab}$ & $11.25 \mathrm{cde}$ \\
$\mathrm{T}_{7} 100 \mathrm{mM}$ proline at seedling stage & $0.082 \mathrm{bc}$ & $0.13 \mathrm{abc}$ & $4.02 \mathrm{~cd}$ & $7.57 \mathrm{ab}$ & $11.59 \mathrm{bcd}$ \\
$\mathrm{T}_{8} 100 \mathrm{mM}$ proline at vegetative stage & $0.10 \mathrm{a}$ & $0.14 \mathrm{ab}$ & $4.91 \mathrm{a}$ & $7.29 \mathrm{~b}$ & $12.20 \mathrm{abc}$ \\
$\mathrm{T}_{9} 10 \mathrm{mM}$ proline at seedling and vegetative stages & $0.08 \mathrm{bc}$ & $0.11 \mathrm{cde}$ & $3.99 \mathrm{~cd}$ & $6.17 \mathrm{c}$ & $10.16 \mathrm{f}$ \\
\hline $\mathrm{SE}( \pm)$ & 0.0034 & 0.0037 & 4.54 & 5.73 & 4.78 \\
$\mathrm{CV}(\%)$ & 13.74 & 8.27 & 0.134 & 0.240 & 0.309 \\
\hline
\end{tabular}

Same letter in a column represents insignificant difference at $\mathrm{p}<0.05$.

$\mathrm{SE}=$ Standard errors of means

$\mathrm{CV}=$ Co-efficient of variation

Table 6. $\mathrm{K}^{+} / \mathrm{Na}^{+}$ratio in grain and straw of $\mathrm{T}$. Aman rice (BR23) influenced by exogenous proline under salinity conditions

\begin{tabular}{|c|c|c|}
\hline \multirow[t]{2}{*}{ Treatment } & \multicolumn{2}{|c|}{$\mathrm{K}^{+} / \mathrm{Na}^{+}$ratio } \\
\hline & Grain & Straw \\
\hline $\mathrm{T}_{0}$ Control & $14.37 \mathrm{~d}$ & $3.710 \mathrm{~d}$ \\
\hline $\mathrm{T}_{1} 25 \mathrm{mM}$ proline at seedling stage & $23.26 \mathrm{ab}$ & $4.62 b c$ \\
\hline $\mathrm{T}_{2} 25 \mathrm{mM}$ proline at vegetative stage & $20.19 \mathrm{c}$ & $6.13 \mathrm{a}$ \\
\hline $\mathrm{T}_{3} 25 \mathrm{mM}$ proline at seedling and vegetative stages & $23.07 \mathrm{bc}$ & $4.90 \mathrm{~b}$ \\
\hline $\mathrm{T}_{4} 50 \mathrm{mM}$ proline at seedling stage & $21.15 b c$ & $4.65 \mathrm{bc}$ \\
\hline $\mathrm{T}_{5} 50 \mathrm{mM}$ proline at vegetative stage & $22.11 b c$ & $6.54 \mathrm{a}$ \\
\hline $\mathrm{T}_{6} 50 \mathrm{mM}$ proline at seedling and vegetative stages & $25.96 \mathrm{a}$ & $4.80 \mathrm{bc}$ \\
\hline $\mathrm{T}_{7} 100 \mathrm{mM}$ proline at seedling stage & $24.04 \mathrm{ab}$ & $5.97 \mathrm{a}$ \\
\hline $\mathrm{T}_{8} 100 \mathrm{mM}$ proline at vegetative stage & $22.11 b c$ & $4.80 \mathrm{bc}$ \\
\hline $\mathrm{T}_{9} 10 \mathrm{mM}$ proline at seedling and vegetative stages & $22.11 \mathrm{bc}$ & $4.22 \mathrm{~cd}$ \\
\hline $\mathrm{SE}( \pm)$ & 0.9693 & 0.2830 \\
\hline $\mathrm{CV}(\%)$ & 7.14 & 7.07 \\
\hline
\end{tabular}

Same letter in a column represents insignificant difference at $\mathrm{p}<0.05$.

$\mathrm{SE}=$ Standard errors of means

$\mathrm{CV}=$ Co-efficient of variation

Salt stress disturbs cytoplasmic $\mathrm{K}^{+} / \mathrm{Na}^{+}$homeostasis, causing an increase in $\mathrm{Na}^{+}$to $\mathrm{K}^{+}$ratio in the cytosol (Zhu, 2003). It has been reported that salt stress causes increased uptake of $\mathrm{Na}^{+}$and $\mathrm{Cl}^{-}$, and decreased uptake of essential cations particularly $\mathrm{K}^{+}$(Khan et al., 2003). Proline has an added advantage under soil salinity as it may lower down Na uptake by plants and increase $\mathrm{K}$ uptake, thereby protecting crops from the detrimental effects of Na. Kaya et al. (2013) showed that sodium concentrations were higher in the tissues of plants grown under saline conditions while compatible solute treatments significantly reduced sodium concentration in the plant tissues. Abd El-Samad et al. (2011) showed that application of Pro increased $\mathrm{K}^{+} / \mathrm{Na}^{+}$ratio in rice plants. Nounjan et al. (2012) on Thai aromatic rice (cv.
KDML105; salt-sensitive) also found the similar result due to exogenous application of Pro.

\section{Conclusion}

Physiological stress due to salinity is the main cause reducing crop yields in coastal saline areas of Bangladesh. Exogenous application of Pro with suitable crop varieties having higher yield potential could contribute to the improvement of crop production in saline condition. Our experiment shows that salinity reduced growth and yield of rice. On the other hand, exogenous Pro increased growth and yield contributing characters of BR-23, resulting higher grain and straw yields of rice. The increased growth and yield of rice by Pro were accompanied with higher $\mathrm{K}^{+} / \mathrm{Na}^{+}$ratio and 
uptake of nutrients. It can be concluded from the present study that Pro confers tolerance to salinity in aman rice due to increasing nutrient uptake, maintaining higher $\mathrm{K}^{+} / \mathrm{Na}^{+}$ratio and probably enhancing antioxidant defense systems. However, further studies are required to find out the beneficial roles of exogenous Pro at reproductive stage under saline conditions.

\section{Acknowledgements}

This work was supported by a grant from the Bangladesh Agricultural Research Council.

\section{References}

Abbas, F.M., Jasim, A.M. and Al-Taha, H.A. 2012. Effect of exogenous proline on protein pattern changes in Citrus sinensis (L.) Osbeck under in vitro salt stress. Advances in Agriculture \& Botanics-International Journal of the Bioflux Society, 4(2): 36-41.

Abd El-Samad, H.M., Shaddad, M.A.K. and Barakat, N. 2011. Improvement of plants salt tolerance by exogenous application of amino acids. Journal of Medicinal Plants Research, 5(24): 5692-5699.

Ali, Q., Ashraf, M. and Athar, H.R. 2007. Exogenously applied proline at different growth stages enhances growth of two maize cultivars grown under water deficit conditions. Pakistan Journal of Botany, 39(4): 1133-1144.

Ashraf, M. and Foolad, M.R. 2007. Roles of glycinebetaine and proline in improving plant abiotic stress tolerance. Environmental and Experimental Botany, 59: 206-216. https://doi.org/10.1016/j.envexpbot.2005.12.006

Azevedo, N. and Sharma, S.K. 2006. Effect of salinity on growth performance and internal distribution of sodium, potassium and chloride in Vicia faba L. Indian Journal of Plant Physiology, 38(1): 69-72.

Banu, M.N.A., Hoque, M.A., Sugimoto-Watanabe, M., Mastuoka, K., Nakamura, Y., Shimoishi, Y. and Murata, Y. 2009. Proline and glycinebetaine induce antioxidant defense gene expression and suppress cell death in cultured tobacco cells under salt stress. Journal of Plant Physiology, 166: 146156. https://doi.10.1016/j.jplph.2008.03.002

Bhusan, D., Das, D.K., Hossain, M., Murata, Y. and Hoque, M.A. 2016. Improvement of salt tolerance in rice (Oryza sativa L.) by increasing antioxidant defense systems using exogenous application of proline. Australian Journal of Crop Science, 10(1): 50-56.

Burritt, D.J., 2012. Proline and the cryopreservation of plant tissues functions and practical applications. In I. Katkov (ed). Current Frontiers in Cryopreservation: IntechOpen. pp. 415-430. Croatia. https://doi.10.5772/36249

Chinnusamy, V., Jagendorf, A. and Zhu, J.K. 2005. Understanding and improving salt tolerance in plants. Crop Science, 45: 437448.

Deivanai, S., Xavier, R., Vinod, V., Timalata, K. and Lim, O.F. 2011. Role of exogenous proline in ameliorating salt stress at early stage in two rice cultivars. Journal of Stress Physiology and Biochemistry, 7(4): 157-174.

Haque, M.A., Jahiruddin, M., Hoque, M.A., Rahman, M.Z. and Clarke, D. 2014: Temporal variability of soil and water salinity and its effect on crop at Kalapara upazila. Journal of Environmental Sciences and Natural Resources, 7(2): 111-114. https://doi.org/10.3329/jesnr.v7i2.22218

Hoque, M.A., Banu, M.N.A., Nakamura, Y., Shimoishi, Y. and Murata, Y. 2008. Proline and glycinebetaine enhance antioxidant defense and methylglyoxal detoxification systems and reduce $\mathrm{NaCl}$-induced damage in cultured tobacco cells. Journal of Plant Physiology, 165: 813-824. https://doi.10.1016/j.jplph.2007.07.013

Hoque, M.A., Okuma, E., Banu, M.N.A., Nakamura, Y., Shimoishi, Y. and Murata, Y. 2007. Exogenous proline mitigates the detrimental effects of salt stress more than exogenous betaine by increasing antioxidant enzyme activities. Journal of Plant Physiology, 164: 553-561. https://doi.10.1016/j.jplph.2006.03.010

Hossain, M.A., Hoque, M.A., Burritt, D.J. and Fujita, M. 2014. Proline protects plants against abiotic oxidative stress: biochemical and molecular mechanisms. In P. Ahmad (ed) Oxidative damage to plants: Elsevier Inc. pp. 477-522. San Diego. https://doi.org/10.1016/B978-0-12-799963-0.00016-2

Islam, M.T., Sharma, P.C., Gautam, R.K., Singh, D., Singh, S., Panesar, B. and Ali, S. 2011. Salt tolerance in parental lines of rice hybrids through physiological attributes and molecular markers. International Journal of Experimental Agriculture, 2(1): 1-7.

Jaleel, C.A., Gopi, R., Manivannan, P. and Panneerselvam, R. 2007. Responses of antioxidant defense system of Catharanthus roseus (L.) G. Don. to paclobutrazol treatment under salinity. Acta Physiologiae Plantarum, 29: 205-209.

Jaleel, C.A., Gopi, R., Manivannan, P. and Panneerselvam, R. 2008. Soil salinity alters the morphology in Catharanthus roseus and its effects on endogenous mineral constituents. EurAsian Journal of BioSciences, 2(2): 18-25.

Khan, A.A., Rao, S.A. and McNeilly, T. 2003. Assessment of salinity tolerance based upon seedling root growth response function in maize (Zea mays L.). Euphytica, 131: 81-89.

Khanam, M., Rahman, M.M., Islam, M.R. and Islam, M.R. 2001. Effect of manures and fertilizers on the growth and yield of BRRI dhan30. Pakistan Journal of Biological Sciences, 4: 172-174.

Kibria, M.G., Hossain, M., Murata, Y. and Hoque, M.A. 2017. Antioxidant defense mechanisms of salinity tolerance in rice genotypes. Rice Science, 24(3): 155-162. https://doi.org/10.1016/j.rsci.2017.05.001

Mahajan, S., and Tuteja, N. 2005. Cold, salinity and drought stresses. An overview. Archives of Biochemistry and Biophysics, 444: 139-158. https://doi.10.1016/j.abb.2005.10.018

Mali, B.S., Thengal, S.S. and Pate, P.N. 2012. Physico-chemical characteristics of salt affected soil from Barhanpur, M S., India. Annals of Biological Research, 3(8): 4091-4093.

Miah, M.A., Panaullah, G.M., Rahman, M.S. and Ishaque, M. 1992: Effect of soil salinity and phosphorus on the growth yield and mineral nutrition of rice. Bangladesh Journal of Rice, 3: $1-5$.

Momayezi, M.R., Zaharah, A.R., Hanafi, M.M. and Ismail, M.R. 2010. Effect of chloride and sulfate salinity on nutrient uptake in Iranian rice (Oryza sativa L.). 19 ${ }^{\text {th }}$ World Congress of Soil Science, Soil solutions for a changing world, Brisbane, pp.36-39.

Nounjan, N., Nghia, P.T. and Theerakulpisut, P. 2012. Exogenous proline and trehalose promote recovery of rice seedlings from salt-stress and differentially modulate antioxidant enzymes and expression of related genes. Journal of Plant Physiology, 169: 596-604. https://doi.10.1016/j.jplph.2012.01.004

Papon, K.D., Murata, Y., Hoque, M.A. and Ali, M.A. 2015. Effect of soil salinity and exogenous proline application on rice growth, yield, biochemical and antioxidant enzyme activities. EC Agriculture, 2: 229-240.

SRDI. 2010. Saline soils of Bangladesh. SRMAF Project, Ministry of Agriculture, Bangladesh, pp.1-60.

Zhu, J.K. 2003: Regulation of ion homeostasis under salt stress. Current Opinion of Plant Biology, 6: 441-445. 